https://helda.helsinki.fi

\title{
Clinical course of H1N1-vaccine-related narcolepsy
}

\section{Sarkanen, Tomi}

2016-03

Sarkanen , T , Alakuijala , A \& Partinen , M 2016, ' Clinical course of H1N1-vaccine-related narcolepsy ' , Sleep Medicine , vol. 19 , pp. 17-22 . https://doi.org/10.1016/j.sleep.2015.11.005

http://hdl.handle.net/10138/224040

https://doi.org/10.1016/j.sleep.2015.11.005

publishedVersion

Downloaded from Helda, University of Helsinki institutional repository.

This is an electronic reprint of the original article.

This reprint may differ from the original in pagination and typographic detail.

Please cite the original version. 
Original Article

\title{
Clinical course of H1N1-vaccine-related narcolepsy
}

\author{
Tomi Sarkanen a,b,c,*, Anniina Alakuijala b,c,d , Markku Partinen b,c \\ a Clinic of Neurology, Central Finland Central Hospital, Jyväskylä, Finland \\ ${ }^{\mathrm{b}}$ Helsinki Sleep Clinic, Vitalmed Research Centre, Helsinki, Finland \\ ${ }^{\mathrm{c}}$ Department of Neurological Sciences, University of Helsinki, Helsinki, Finland \\ ${ }^{d}$ Department of Clinical Neurophysiology, HUS Medical Imaging Center, Helsinki University Central Hospital, Finland
}

\section{A R T I C L E I N F O}

\section{Article history:}

Received 8 July 2015

Received in revised form 16 October 2015

Accepted 9 November 2015

Available online 25 November 2015

\section{Keywords:}

Narcolepsy

Cataplexy

Vaccine

Pandemrix

\begin{abstract}
A B S T R A C T
Objective: To follow and analyze the clinical course and quality of life of Pandemrix H1N1-vaccinerelated narcolepsy (pNT1).

Methods: Twenty-six drug-naïve confirmed pNT1 subjects completed Epworth Sleepiness Scale (ESS), Ullanlinna Narcolepsy Scale (UNS), Swiss Narcolepsy Scale (SNS), Rimon's Brief Depression scale (RDS), and WHO-5 Well-being index questionnaires near the disease onset and in a follow-up a minimum of two years later. The number of cataplexies and body mass index (BMI) were recorded. The effects of hypocretin-1 levels and sleep recording results were analyzed. The findings at the follow-up visit were compared with 25 non-vaccine-related type 1 narcolepsy (NT1) subjects.

Results: In pNT1, RDS score decreased significantly (mean 10.2, SD 4.7 vs mean 6.7, SD 4.5, $p=0.003$ ). Median of BMI increased from $20.8 \mathrm{~kg} \mathrm{~m}^{-2}$ to $23.4 \mathrm{~kg} \mathrm{~m}^{-2}, p<0.001$. There were no significant differences in other sleep scores. However, deviation and range in questionnaire scores at the follow-up were wide. Subjects with very low or undetectable hypocretin-1 levels had worse scores in UNS (mean 26.4, SD 6.95 vs mean 19.1, SD 3.83, $p=0.006$ ) and ESS (mean 17.9, $\mathrm{SD}=4.29$ vs mean $14.1, \mathrm{SD}=3.70, p=0.047$ ) than those with hypocretin-1 levels of $20-110 \mathrm{pg} / \mathrm{mL}$. Most disabling symptoms were excessive daytime sleepiness and disturbed sleep. There were no significant differences between the scores in pNT1 and NT1.

Conclusions: Clinical course of pNT1 is heterogeneous but the evolution of pNT1 seems similar to NT1. Lower hypocretin levels in pNT1 are associated with a more severe phenotype.
\end{abstract}

(c) 2015 Elsevier B.V. All rights reserved.

\section{Introduction}

Narcolepsy type 1 is a central disorder of hypersomnolence characterized by a loss of hypocretin- (orexin-) producing neurons in the hypothalamus. According to the current hypothesis, narcolepsy type 1 is an autoimmune disease, although the specific mechanism remains elusive [1-3]. The main symptoms of narcolepsy are excessive daytime sleepiness, disturbed nocturnal sleep, cataplexy, sleep paralyses, and hallucinations during sleep-wake transitions. Other common associated features are increased weight, precocious puberty, behavioral problems, and psychiatric comorbidity [4-6]. There is a great heterogeneity in symptoms as some subjects are severely affected and disabled, whereas others manage without medication.

The incidence of narcolepsy increased significantly in countries where AS03-adjuvanted Pandemrix H1N1 vaccination was used

\footnotetext{
* Corresponding author. Clinic of Neurology, Central Finland Central Hospital, Keskussairaalantie 19, 40620 Jyväskylä, Finland. Tel.: +358 40967 7345; fax: +358 9440260.

E-mail address: tomi.sarkanen@ksshp.fi (T. Sarkanen).
}

widely in 2009-2010 [5,7-11]. The increase was first noted in children and adolescents and later, to a lesser degree, in adults. However, as previously reported, diagnostic delay in narcolepsy has been rather long, over 10 years in many cases, although a shorter delay, especially in younger age groups, has also been observed [12,13]. In Pandemrix H1N1-vaccine-related narcolepsy type 1 (pNT1) cases, the diagnostic delay has been much shorter. Reasons for this remain somewhat unclear. A more sudden onset and severe symptoms in pNT1 than in sporadic narcolepsy type 1 (NT1) could be an explaining factor $[5,6]$. On the other hand, a comparison between Finnish children with pNT1 and Italian children with NT1 appears to show similar clinical pictures, although subjects with pNT1 often had increased nocturnal sleep disturbance [14]. In a recent study on polysomnographic and actigraphic characteristics of pNT1 and NT1, we found that NT1 subjects had delayed sleep-wake rhythms compared to pNT1, but otherwise we did not see dramatic differences [15].

As pNT1 is considered a drug-adverse effect, the countries where Pandemrix was used are now paying monetary compensations to the affected patients. Compensation policy varies. Considering the heterogeneous disease course as described above, determining the long-term (or even short-term) handicap caused by narcolepsy is 
challenging, and the suitable monetary compensation is difficult to determine if it is based on individual consideration. Nonetheless, narcolepsy causes marked social and economical burden for patients and their close relatives, in addition to deleterious effects which may occur from health-related reductions in quality of life [16,17].

The loss of hypocretin might not be the only biological factor contributing to the symptoms of narcolepsy. Different compensatory mechanisms for the loss of function of hypocretin - such as increase in histamine neurons - are probably also involved [18-21]. Furthermore, we have recently found that narcolepsy patients have autoantibodies that stain distinct cell populations in rat brain, namely melanin-concentrating hormone $(\mathrm{MCH})$, proopiomelanocortin (POMC), and neuropeptide glutamic acidisoleucine/alpha-melanocyte-stimulating hormone (NEI/ $\alpha \mathrm{MSH})[22]$. In addition, $\mathrm{MCH} / \mathrm{POMC}$ injections in rat brains were found to be associated with disturbed sleep patterns. Recently, Heier et al. also reported changes in nerve cell biomarkers in cerebrospinal fluid (CSF) of narcoleptic patients [23]. These biological alterations, shortened diagnostic delay, abrupt onset, and social and economical factors make it important to follow and analyze the clinical course of pNT1.

\section{Methods}

\subsection{Subjects}

Twenty-six pNT1 subjects (15 male, 11 female) from Helsinki Sleep Clinic, Vitalmed Research Centre were recruited for this study. They completed a self-administered modified Basic Nordic Sleep Questionnaire (mBNSQ) near the onset of the disease and at the follow-up visit at least two years later. Follow-up results were also compared with 25 (10 male, 15 female) NT1 subjects. All subjects had confirmed type 1 narcolepsy. Hypocretin-1 was measured in 21 subjects in pNT1 group and in 18 subjects in NT1 and was below $110 \mathrm{pg} / \mathrm{mL}$ in all cases. When hypocretin-1 was not measured, the subjects had unambiguous cataplexy, were HLA DQB1*06:02 positive, and had a positive multiple sleep latency test (MSLT) for narcolepsy (sleep latency less than eight minutes and two or more sleep onset REM sleep periods [SOREMPs]). Hypocretin levels were measured in Rinnekoti Research Laboratory using Human orexin-A RIA Kit (Phoenix Pharmaceutical, Inc., Belmont, CA) with Stanford reference sample. Characteristics of study subjects are listed in Table 1.

Classification as pNT1 was done if the subject had had confirmed Pandemrix vaccination during winter 2009-2010 and symptoms of narcolepsy started within 2 years after vaccination. Most sporadic narcolepsy type 1 subjects had their disease onset before $\mathrm{a}(\mathrm{H} 1 \mathrm{~N} 1) \mathrm{pdm} 09$ pandemic. Four subjects had their onset during 2009 or 2010 . None of them were vaccinated before symptom onset or had influenza like illness (ILI). Ten were vaccinated with Pandemrix after the disease onset. Vaccination data was missing for three subjects. They all had the disease onset years before vaccination.

Subjects were drug-naïve at their first visit, ie, they did not have any narcolepsy medication including sodium oxybate, stimulants, selective serotonin, or serotonin and norepinephrine reuptake inhibitors. The treating neurologist/sleep expert made following treatment decisions independently of this study.

\subsection{Questionnaires and measures}

mBNSQ includes multiple sub-scales and standardized tools for assessment of sleep-related symptoms [24]. Questionnaires used in this study were Epworth Sleepiness Scale (ESS), Ullanlinna Narcolepsy Scale (UNS), Swiss Narcolepsy Scale (SNS), WHO-5 WellBeing Scale (WHO5), and Rimon's Brief Depression Scale (RDS) [24-27]. A separate questionnaire for disability caused by the disease was also used. This questionnaire included main symptoms of narcolepsy and disability caused by them on scale zero (subject does not have this symptom) to four (severe disability). Weight and height were asked to calculate body mass index (BMI). Number of cataplectic attacks per week was asked (CPL/week). If questionnaires were partially completed, one follow-up phone call was made or a new questionnaire was sent.

\subsection{Response rate}

Initial complete response rate for all questionnaires combined was $61 \%$ and partial response rate was $89 \%$ (each measure or questionnaire analyzed separately). After follow-up phone calls or new questionnaires, response rates were $81 \%$ and $96 \%$, respectively.

\subsection{Statistical analyses}

Statistical analyses were performed using SPSS (IBM SPSS® Statistics 19.0, Armonk, NY, USA) and STATA version 13.1 (Stata Corporation, TX). Graphs were made using R software (R development core team, 2014) and ggplot2 package (H. Wickham. ggplot2: elegant graphics for data analysis, Springer New York, 2009) or Excel. For statistical comparisons, parametric and non-parametric methods were used according to the normality of distributions, which were analyzed using Kolmogorov-Smirnov and Shapiro-Wilkinson tests and by calculating skewness and kurtosis of continuous variables. Mean (M) and standard deviation (SD) are reported for variables following normal distribution and median (Mdn) and range for nonparametrically distributed variables.

Table 1

Characteristics of study subjects.

\begin{tabular}{|c|c|c|}
\hline & pNT1 & NT1 \\
\hline Female & $11 / 26(42 \%)$ & $15 / 25(60 \%)$ \\
\hline Age at onset, years, median (range) & $15.2(6.0-46.5)$ & $14.5(11.6-39.0)$ \\
\hline Age at diagnosis, years, median (range) & $15.9(6.4-48.5)$ & $18.5(11.6-39.0)^{*}$ \\
\hline Diagnostic delay, years, mean (SD) & $1.1(0.9)$ & $6.6(7.6)^{\dagger}$ \\
\hline Age at follow-up visit, mean (SD) & $18.5(8.2-49.9)$ & $28.2(12.7-51.9)^{* * *}$ \\
\hline Disease duration at follow-up visit, years, median, (range) & $3.3(2.1-4.6)$ & $10.2(2.3-31.4)^{* *}$ \\
\hline Vaccination to disease onset, days, median, (range) & $145(6-487)$ & NA \\
\hline Hypocretin-1, pg/mL, median (range) & $8(0-108)$ & $20(0-79)$ \\
\hline MSLT sleep latency, minutes, median (range) & $2.25(0.4-10.0)$ & $3.10(0.1-6.9)$ \\
\hline SOREMPs, median (range) & $3(0-5)$ & $3(0-5)$ \\
\hline
\end{tabular}

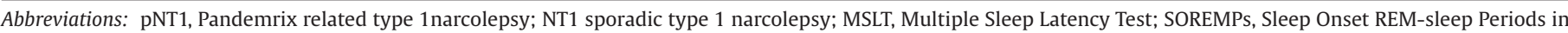
MSLT; NA, Not applicable; Hypocretin-1 measured in 19/24 Pandemrix H1N1-vaccine-related subjects and in 15/23 sporadic cases.

* Mann-Whitney $\mathrm{U}, p<0.05$; $^{* *}$ Mann-Whitney $\mathrm{U}, p<0.005$; $^{\dagger}$ Independent samples $t$-test $p<0.005$. 
Table 2

Symptoms of Pandemrix H1N1-vaccine-related narcolepsy at first and follow-up visits.

\begin{tabular}{|c|c|c|c|c|}
\hline & First visit & Second visit & 95\% CI for Mean difference & $p$ \\
\hline ESS, mean $(S D)$ & $15.8(6.2)$ & $14.9(5.0)$ & $-3.3,2,0$ & 0.631 \\
\hline UNS, mean (SD) & $23.3(7.3)$ & $21.1(6.9)$ & $-5.3,1.9$ & 0.352 \\
\hline SNS, mean $(S D)$ & $-40.4(38.3)$ & $-32.0(29.7)$ & $-9.8,25.6$ & 0.368 \\
\hline RDS, mean (SD) & $10.2(4.7)$ & $6.7(4.5)$ & $-5.5,-1.3$ & 0.003 \\
\hline WHO5, mean $(S D)$ & $45.5(24.8)$ & $48.0(19.3)$ & $-10.4,15.4$ & 0.688 \\
\hline CPL / week, median (range) & $15(0-210)$ & $10.5(0-210)$ & NA & 0.281 \\
\hline BMI, kgm-2, median (range) & $20.8(14.4-35.1)$ & $23.4(16.6-38.3)$ & NA & $<0.001$ \\
\hline
\end{tabular}

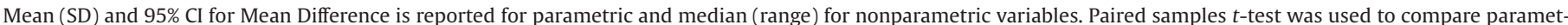
ric and Wilcoxon signed rank test to compare nonparametric variables between different visits in pNT1.

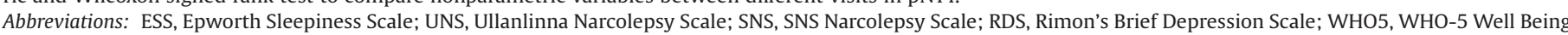
Scale; BMI, Body Mass Index; CPL/week, cataplexies per week; NA, Not applicable (nonparametric variables). Statistically significant differences are written in bold.

\subsection{Ethics}

The study was approved by the Helsinki and Uusimaa hospital district ethical committee. Written informed consent was obtained from adult subjects and in children and adolescents from themselves and their parents.

\section{Results}

Age at onset of narcolepsy was similar in pNT1 and NT1 (Table 1). Diagnostic delay was significantly shorter in pNT1. The follow-up visit was done earlier in the disease course in pNT1 than in NT1 and, therefore, pNT1 subjects were younger at the follow-up. There were no significant differences in CSF hypocretin-1 levels or median sleep latencies in MSLT between the groups.

\subsection{Follow-up of pNT1}

A paired-samples $t$-test was conducted to compare questionnaire results between the two visits in pNT1 (Table 2). In general, mean differences in scores were rather low: ESS -0.63 points, UNS -1.68 points, SNS 7.88 points, and WHO5 2.54 points. Mean difference in RDS was an exception as the reduction was 3.44 points and was the only statistically significant change, $t(24)=0.381, p=0.003$, $d=0.67$. However, standard deviations in changes in scores were rather wide which implies that there were patients who were clearly worse or had their symptoms markedly ameliorated on follow-up visit (Fig. 1). Wilcoxon Signed-ranks test was used to compare differences in BMI and CPL/week. Median of BMI increased $2.3 \mathrm{~kg} \mathrm{~m}^{-2}$, $Z=3.8, p<0.001$. Amount of weekly cataplexies did not change significantly.

\subsection{Effect of CSF hypocretin levels and MSLT}

Linear regression analysis was conducted to find out whether CSF hypocretin levels, sleep latency in MSLT, or number of SOREMPs in MSLT explained changes in sleep questionnaire scores. We did not find any model based on these variables that would explain the changes (data not shown). We then divided study subjects into two subgroups based on hypocretin levels: those with very low $(<20 \mathrm{pg} /$ $\mathrm{mL})$ or undetectable hypocretin levels $(N=13$ in pNT1 and $N=9$ in NT1) and those with hypocretin levels of $20-110 \mathrm{pg} / \mathrm{mL}(N=8$ in pNT1 and $N=9$ in NT1). A cut-off limit of 20 was used because the assay is not absolutely accurate below $20 \mathrm{pg} / \mathrm{mL}$, although we had specific numeric values also for these results. Here, in pNT1 group, subjects who had very low hypocretin levels had higher UNS scores $(\mathrm{M}=24.4, \mathrm{SD}=6.64)$ than subjects with higher hypocretin levels $(\mathrm{M}=18.8, \mathrm{SD}=4.46), t(19)=-3.1, p=0.048)$ (Fig. 2$)$. They also had higher ESS scores $(\mathrm{M}=17.2, \mathrm{SD}=4.64$ vs $\mathrm{M}=13.1, \mathrm{SD}=1.87)$, $t(18)=-2.1, p=0.040$ (Fig. 3). Furthermore, diagnostic delay was shorter in patients with very low hypocretin $(M=207$ days, $S D=161)$ compared with hypocretin levels of $20-110 \mathrm{pg} / \mathrm{mL}(\mathrm{M}=803$ days, $\mathrm{SD}=257), t(17)=-6.2, p<0.005$. We did not see differences in scores at the first visit or mean differences between visits by hypocretin levels. Neither did we find these differences in NT1 (data not shown).

We also analyzed the relationship between separate UNS items and hypocretin status. Those pNT1 subjects who had hypocretin-1 below $20 \mathrm{pg} / \mathrm{mL}$ experienced head nods more often than those with higher levels ( $\mathrm{Mdn}=4$ [daily or almost daily], $\mathrm{M}=3.18,95 \% \mathrm{CI} 2.24-$ 4.12 vs $\mathrm{Mdn}=1.5$ [monthly to less than monthly], $\mathrm{M}=1.63,95 \% \mathrm{CI}$ $0.29-2.96, p=0.033$ ) on the follow-up visit. There were no differences in other UNS cataplexy items (knee sagging, mouth opening or falling to the ground).
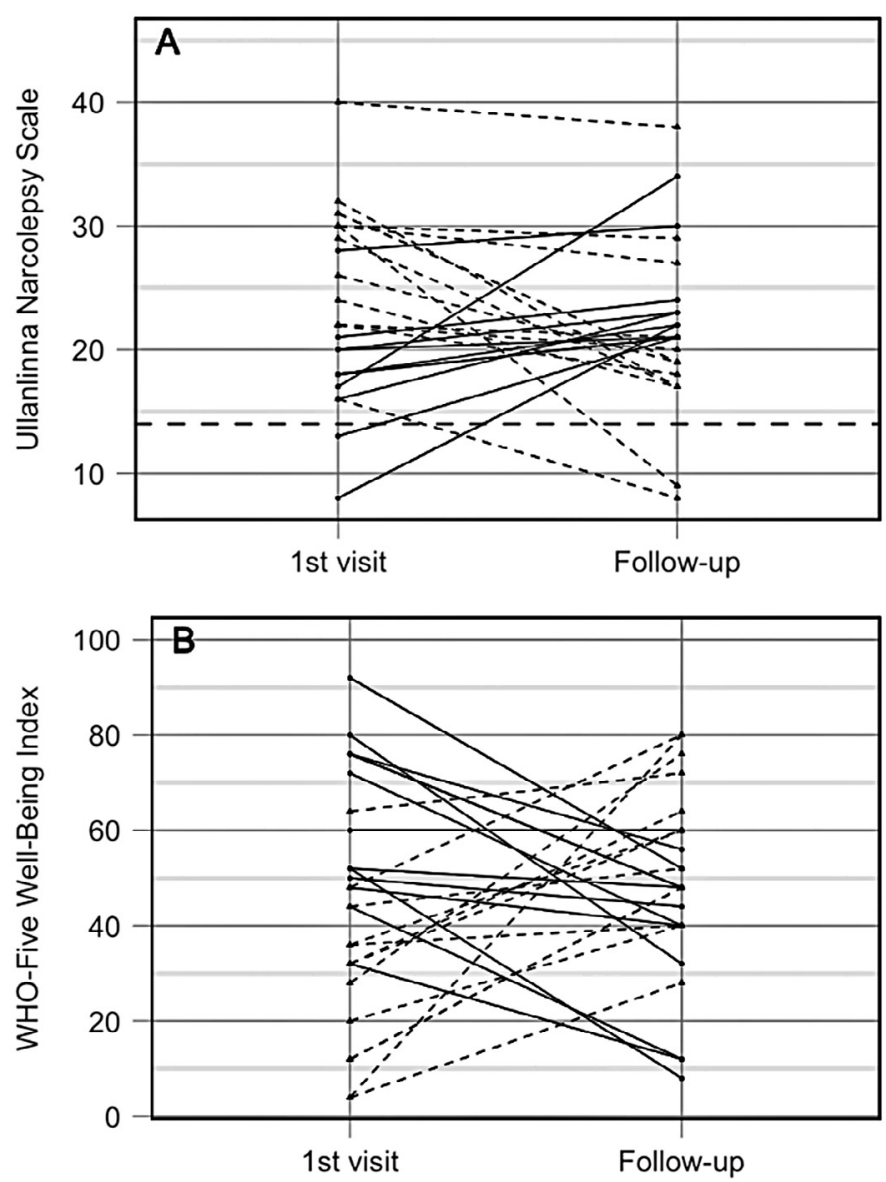

Fig. 1. Changes in Ullanlinna Narcolepsy Scale (a) and WHO-5 Well-Being index (b) between visits in Pandemrix related narcolepsy. Dotted line, Ullanlinna Narcolepsy Scale cut point of 14 points; solid line with dots, worse or no change; dotted line with triangles: better. 


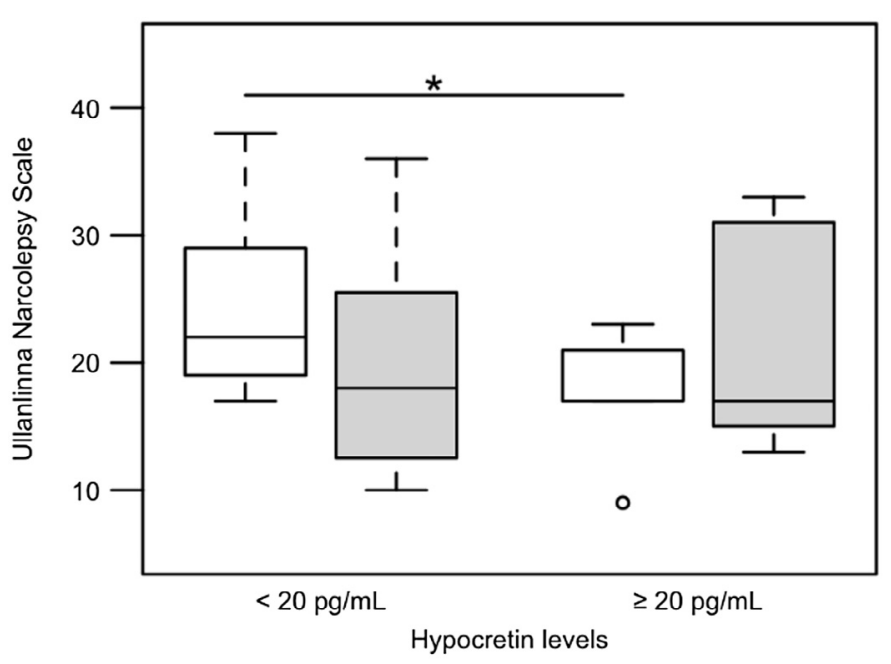

Fig. 2. Ullanlinna Narcolepsy Scale on follow-up visit based on hypocretin levels. White boxplot, Pandemrix H1N1-vaccine-related narcolepsy; gray boxplot, sporadic narcolepsy; asterisk, independent samples $t$-test, ${ }^{*} p<0.05$.

\subsection{Effect of age}

Eight post-Pandemrix cases were adults and 18 were children or adolescents ( $<18$ years) at the first visit. At the second visit, the corresponding figures were 14 adults and 12 under-aged. In the sporadic narcolepsy group, 18 , also a majority of subjects, had their disease onset as a child or an adolescent. Only three of them were under-aged at the study visit. In the pNT1 group, there were no statistically significant differences between the adult and underaged subjects in sleep questionnaire scores at the first or followup visits. The age of onset correlated strongly with mean sleep latency $(r=0.560, p=0.005)$ and SOREMPs $(r=-0.482, p=0.020)$ in MSLT, but not with the sleep questionnaire scores.

\subsection{Disability caused by narcolepsy}

The most disabling symptom on follow-up visit was daytime sleepiness, as $84 \%$ of subjects reported it causing moderate to severe harm or disability (Fig. 4). Disturbed sleep caused similar harm to

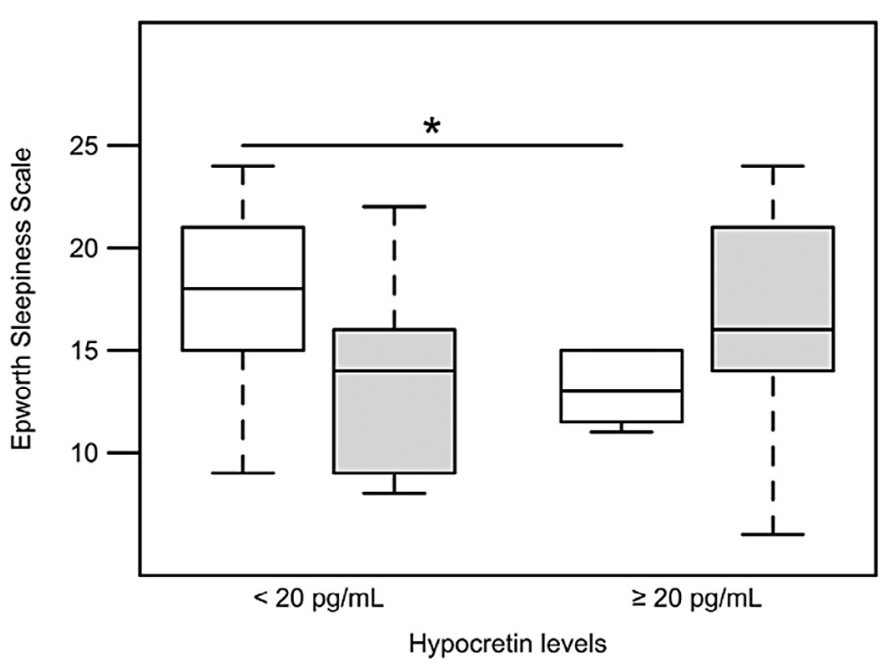

Fig. 3. Epworth Sleepiness Scale on follow-up visit based on hypocretin levels. White boxplot, Pandemrix H1N1-vaccine-related narcolepsy; gray boxplot, sporadic narcolepsy.; asterisk, independent samples $t$-test, ${ }^{*} p<0.05$.
$63 \%$ of subjects. Corresponding figures for partial cataplectic attacks were $37 \%$, generalized cataplectic attacks $32 \%$, hypnagogic or hypnopompic hallucinations $26 \%$, other hallucinations $16 \%$, sleep paralyses $16 \%$, weight increase $21 \%$, and medication adverse effects $27 \%$.

\subsection{Comparison between pNT1 and NT1}

Mann-Whitney U-test showed higher BMI in NT1 ( $M d n=26.4$, range 20.2-47.5) than pNT1 subjects $(\mathrm{Mdn}=23.3$, range 16.6 38.3), $U$ 166.0, $p=0.02$. Cataplexy was more frequent in pNT1 $(\mathrm{Mdn}=10.5$, range $0-210)$ than in NT1 $(\mathrm{Mdn}=3.0$, range $0-50)$, $\mathrm{U}=457.0, p=0.018$. UNS, ESS, SNS, WHO5, and RDS scores were similar and there were no statistically significant differences. Three out of ten vaccinated NT1 subjects had clinical worsening of cataplexy after a(H1N1)pdm09 vaccination.

\subsection{Used medications}

Subjects assessed on the first visit were drug-naïve. Medication on the second visit is listed in Table 3. Ten subjects had both modafinil and sodium oxybate and two were still without any medication. Those two patients had rather mediocre difficulty of symptoms and there were no changes between visits. As our study was not a randomized controlled study, it is not possible to truly evaluate the effect of medication on clinical course of narcolepsy. In our study sample, different medications or their combinations did not have significant effect on sleep scores (data not shown).

\section{Discussion}

pNT1 developed quickly and the diagnostic delay was very short compared to previously reported delays, suggesting more severe clinical phenotype. Our study is the first longitudinal follow-up study of pNT1 and, as far as we know, also the first longitudinal follow-up study of patients with NT1 according to the new diagnostic classification. Our results show that the clinical course of type 1 post-Pandemrix narcolepsy is very variable. Symptoms of most of our patients had ameliorated at least a little at the follow-up visit, but there were few patients who were clearly worse. This seems to be in line with previous reports in NT1 [28].

We did not see any differences in mean sleep scale scores between pNT1 a few years after the disease onset and NT1 who were, however, examined later. This finding suggests that the phenotype of these diseases and their clinical course is similar. Therefore, it might be possible to estimate prognosis of pNT1 based on prior knowledge on evolution of NT1. The finding is important especially in pNT1 compensation issues. For example, a clinician can be inquired about the employment possibilities and disability caused by narcolepsy when an adolescent narcoleptic patient turns 18 years old, or an adult patient seeks disability pension or reimbursement from vaccination adverse effect.

The classic tetrad of narcolepsy symptoms includes excessive daytime sleepiness, cataplexy, hypnagogic and hypnopompic hallucinations, and sleep paralysis. As showed in our study, disturbed nocturnal sleep is also a common and clearly disabling symptom. It is known that treatment of disturbed nocturnal sleep (by, eg, sodium oxybate or melatonin when appropriate) may also improve daytime alertness and other daytime symptoms [29].

Higher UNS and ESS scores on the follow-up visit in subjects with undetectable or very low hypocretin levels compared to those who had a higher amount of hypocretin in CSF is intriguing. This implies that severity of hypocretin deficiency can have an effect on the 


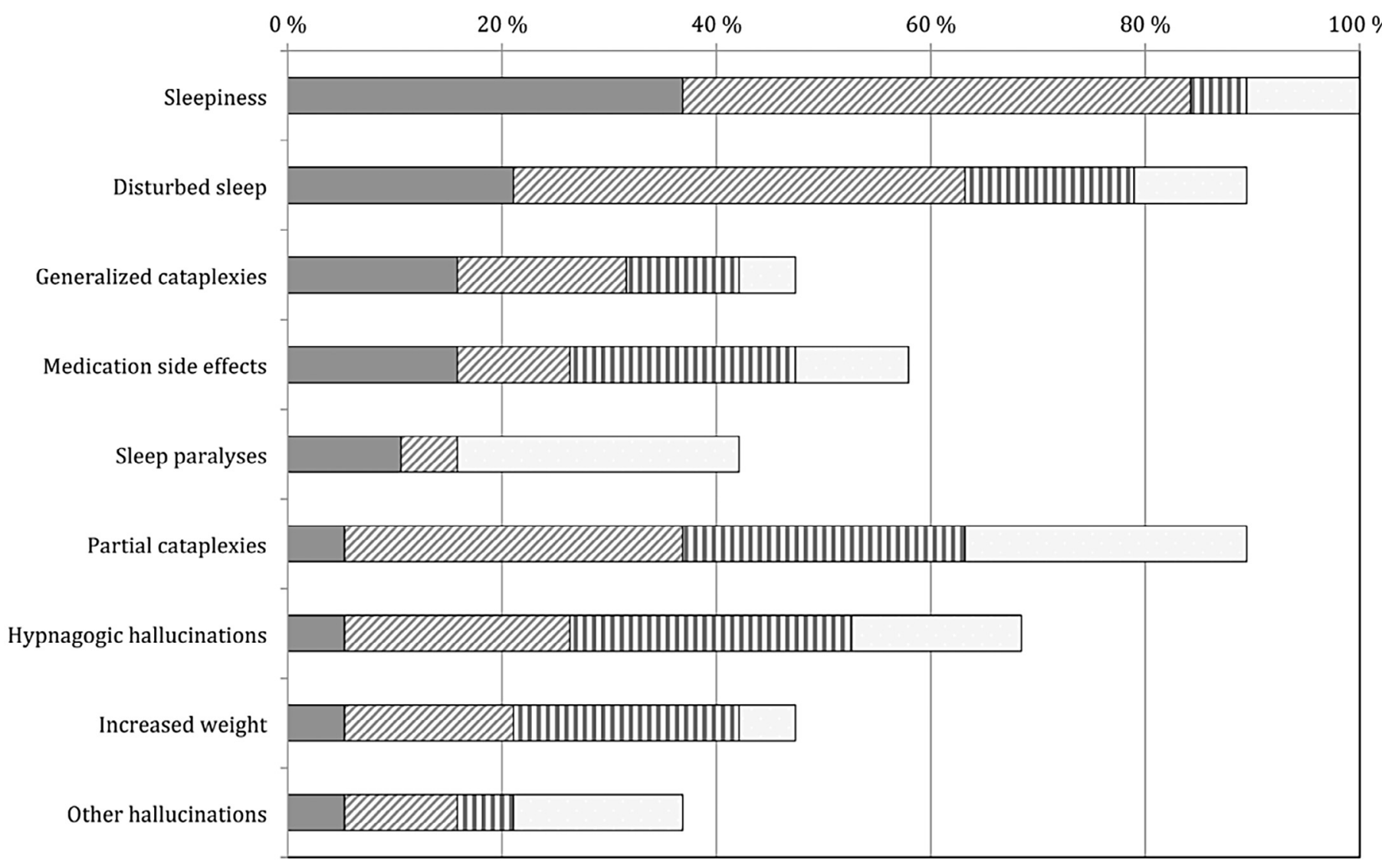

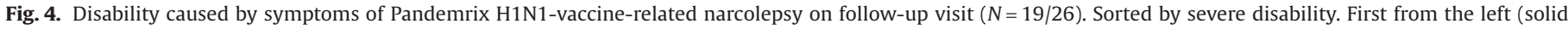

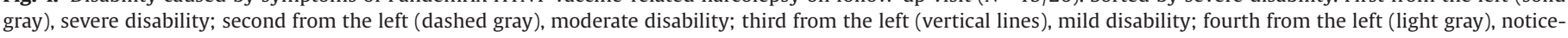
able, no disability.

disease course. Previously, Baumann and coworkers did find more severe MSLT results in patients with undetectable hypocretin levels compared to patients with low, intermediate, or normal hypocretin levels [30]. Conversely, they did not find differences in the same sleep questionnaire scores (UNS, SNS, ESS) that we had used, but their hypocretin detection limit was $60 \mathrm{pg} / \mathrm{mL}$ while in our study it was $20 \mathrm{pg} / \mathrm{mL}$. It is worth noting that our study demonstrated that there was no strict direct relationship between clinical course or symptom severity and CSF hypocretin levels or MSLT findings. For example, we had patients who had very mild symptoms but still undetectable hypocretin levels. Furthermore, those patients whose hypocretin levels were near $110 \mathrm{pg} / \mathrm{mL}$ could have severe symptoms as well.

Lack of direct correlation of hypocretin levels on symptoms implicates also that other neural networks than solely hypocretin are contributing to symptom severity in narcolepsy. Possibilities are, eg, NEI/ $\alpha M S H$ and histamine $[18,20-22]$. It has been shown that there is an inverse relationship between dynamics in histamine and

Table 3

Medication used on follow-up visit.

\begin{tabular}{lr}
\hline Modafinil & 16 \\
Sodium oxybate & 12 \\
Methylphenidate & 3 \\
Venlafaxine & 3 \\
Clomipramine & 2 \\
MAO-B-inhibitors & 1 \\
Other narcolepsy medications (eg, SSRI) & 3 \\
Other medications (incl. melatonin) & 8 \\
No medication & 2 \\
\hline
\end{tabular}

Abbreviations: MAO-B, monoamineoxidase; SSRI, selective serotonin reuptake inhibitor. hypocretin levels [19]. Conversely, hypocretin neurons in the perifornical hypothalamus of rodents strongly excite histaminergic tuberomamillary nucleus neurons through hypocretin-2 receptors. In patients with narcolepsy, histamine may oppose the effects of hypocretin loss. Accordingly, one promising group of medications for narcolepsy are histamine $\mathrm{H} 3$ receptor inverse agonists such as pitolisant [31]. The patients with the worst symptoms may be those whose histamine system, in addition to the hypocretin system, is also defective or not compensating sufficiently.

It has been previously reported that obesity is very common in narcolepsy [4]. BMI increase was clear also in our study while the majority of the subjects still stayed within the normal range. BMI was even higher in NT1 which may also be explained by greater age and longer disease duration of NT1 subjects. Still, reasons for the weight increase remain unknown. It may be due to changes in calorie uptake or expenditure, medication side-effects, or altered metabolic status. As some narcolepsy medications are weight increasing (eg, venlafaxine, clomipramine) and some weight decreasing (sodium oxybate), this is an important aspect to take into account, when considering treatment options.

Somewhat surprisingly, we did not see differences in questionnaire scores between adults and younger subjects. Some previous reports have suggested that childhood narcolepsy presents a distinct clinical picture and with specific symptoms such as facial or generalized hypotonia and increased total sleep time, that gradually ameliorate during the following years [28]. Our questionnaire might not be sensitive enough to separate these symptoms as they are not designed and validated for such purpose. Our adult cases might also have more severe disease than previous sporadic narcolepsy patients in general, since their diagnostic delay has been 
rather short. Previously, diagnostic delay of more than 10 years has been seen. Type 2 error is also possible due to our relatively small sample size.

Our study has some other limitations as well. The study was conducted in a tertiary sleep clinic, which may cause selection bias. Our study population might be more severely affected than most narcoleptic patients as some patients with mild symptoms may have visited our clinic only for diagnostic reasons and the follow-up might have been done in secondary or primary healthcare institutions. Frequent complaints about medication in our study could support this hypothesis. However, we also had some patients without medication on the follow-up visit. Despite these limitations, we are confident that these results can be generalized to pNT1.

Unfortunately, we were not able to perform hypocretin measurements for all the patients, but their diagnosis was still solid and they were most likely hypocretin deficient. They were all HLADQB1*06:02 positive and they all had unambiguous cataplexy and two or more SOREMPs and sleep latency of less than 8 min in MSLT. Theoretically, it could have been interesting to also follow hypocretin levels on second lumbar puncture, but as it would not have had any effect on treatment, we decided not to do it for ethical reasons. Sleep recording follow-up would have been interesting but was not financially possible and not real-life practice either. Moreover, patientreported outcomes are most important in following the treatment effect and symptoms of narcolepsy in real-life settings [32].

In summary, the clinical course of post-Pandemrix narcolepsy is very heterogeneous. Most patients get at least somewhat better at two years' follow up. Improvement in depression scale points could reflect adaptation to disease symptoms. Weight gain is common also in Pandemrix H1N1-vaccine-related narcolepsy. MSLT findings do not correlate very well with symptom severity but lower hypocretin levels are associated with a more severe phenotype on follow-up visit. Therefore, the severity of hypocretin deficiency can have some effect on clinical course of narcolepsy. There is no marked difference in symptoms between sporadic or Pandemrix-associated narcolepsy after two or more years after disease onset.

\section{Conflict of interest}

Dr Sarkanen reports grants from Academy of Finland, during the conduct of the study; non-financial support from UCB Pharma, nonfinancial support from Bioprojet, outside the submitted work. Dr Partinen reports grants from Academy of Finland, during the conduct of the study; personal fees from UCB Pharma, personal fees from Leiras Takeda, personal fees from MSD, personal fees from Cephalon, outside the submitted work. Dr Alakuijala reports grants from Academy of Finland, during the conduct of the study.

The ICMJE Uniform Disclosure Form for Potential Conflicts of Interest associated with this article can be viewed by clicking on the following link: http://dx.doi.org/10.1016/j.sleep.2015.11.005.

\section{Acknowledgements}

We are grateful to all of our patients and their parents who participated in the study.

We thank Anne Huutoniemi, Raili Reemets and all study personnel for their valuable help in organizing and collecting data. We thank Eric Burns for language editing. This study is supported by Academy of Finland grant 260603 (NarpaNord).

\section{References}

[1] Partinen M, Kornum BR, Plazzi G, et al. Narcolepsy as an autoimmune disease: the role of H1N1 infection and vaccination. Lancet Neurol 2014;13:600-13.

[2] Julkunen I, Partinen M. Neuroimmunology: disease mechanisms in narcolepsy remain elusive. Nat Rev Neurol 2014;10:616-17.

[3] Liblau RS, Vassalli A, Seifinejad A, et al. Hypocretin (orexin) biology and the pathophysiology of narcolepsy with cataplexy. Lancet Neurol 2015;14:318-28.

[4] Poli F, Pizza F, Mignot E, et al. High prevalence of precocious puberty and obesity in childhood narcolepsy with cataplexy. Sleep 2013;36:175-81.

[5] Partinen M, Saarenpaa-Heikkila O, Ilveskoski I, et al. Increased incidence and clinical picture of childhood narcolepsy following the 2009 H1N1 pandemic vaccination campaign in Finland. PLoS ONE 2012;7:e33723.

[6] Szakács A, Hallböök T, Tideman P, et al. Psychiatric comorbidity and cognitive profile in children with narcolepsy with or without association to the H1N1 influenza vaccination. Sleep 2015;38:615-21.

[7] Szakács A, Darin N, Hallböök T. Increased childhood incidence of narcolepsy in western Sweden after H1N1 influenza vaccination. Neurology 2013;80:131521.

[8] Heier MS, Gautvik KM, Wannag E, et al. Incidence of narcolepsy in Norwegian children and adolescents after vaccination against H1N1 influenza A. Sleep Med 2013;14:867-71.

[9] Miller E, Andrews N, Stellitano L, et al. Risk of narcolepsy in children and young people receiving AS03 adjuvanted pandemic A/H1N1 2009 influenza vaccine: retrospective analysis. BMJ 2013;346:f794.

[10] Dauvilliers Y, Montplaisir J, Cochen V, et al. Post-H1N1 narcolepsy-cataplexy. Sleep 2010;33:1428-30.

[11] O'Flanagan D, Barret AS, Foley M, et al. Investigation of an association between onset of narcolepsy and vaccination with pandemic influenza vaccine, Ireland April 2009-December 2010. Euro Surveill 2014;19:15-25.

[12] Thorpy MJ, Krieger AC. Delayed diagnosis of narcolepsy: characterization and impact. Sleep Med 2014;15:502-7.

[13] Dauvilliers Y, Arnulf I, Mignot E. Narcolepsy with cataplexy. Lancet 2007;369:499-511.

[14] Pizza F, Peltola H, Sarkanen T, et al. Childhood narcolepsy with cataplexy: comparison between post-H1N1 vaccination and sporadic cases. Sleep Med 2013;15(2):262-5

[15] Alakuijala A, Sarkanen T, Partinen M. Polysomnographic and actigraphic characteristics of patients with H1N1-vaccine-related and sporadic narcolepsy. Sleep Med 2015;16:39-44.

[16] Jennum P, Ibsen R, Petersen ER, et al. Health, social, and economic consequences of narcolepsy: a controlled national study evaluating the societal effect on patients and their partners. Sleep Med 2012;13:1086-93.

[17] Ingravallo F, Gnucci V, Pizza F, et al. The burden of narcolepsy with cataplexy: how disease history and clinical features influence socio-economic outcomes. Sleep Med 2012;13:1293-300.

[18] Saper CB. Restoration: potential for compensatory changes in numbers of neurons in adult human brain. Ann Neurol 2013;74:762-4.

[19] Panula P, Sundvik M, Karlstedt K. Developmental roles of brain histamine. Trends Neurosci 2014;37:159-68.

[20] John J, Thannickal TC, McGregor R, et al. Greatly increased numbers of histamine cells in human narcolepsy with cataplexy. Ann Neurol 2013;74(6):786-93.

[21] Valko PO, Gavrilov YV, Yamamoto M, et al. Increase of histaminergic tuberomammillary neurons in narcolepsy. Ann Neurol 2013;74:794-804.

[22] Bergman P, Adori C, Vas S, et al. Narcolepsy patients have antibodies that stain distinct cell populations in rat brain and influence sleep patterns. Proc Natl Acad Sci U S A 2014;111:E3735-44

[23] Heier MS, Skinningsrud A, Paus E, et al. Increased cerebrospinal fluid levels of nerve cell biomarkers in narcolepsy with cataplexy. Sleep Med 2014;15:614-18.

[24] Partinen M, Gislason T. Basic Nordic Sleep Questionnaire (BNSQ): a quantitated measure of subjective sleep complaints. J Sleep Res 1995;4:150-5.

[25] Hublin C, Kaprio J, Partinen M, et al. The Ullanlinna Narcolepsy Scale: validation of a measure of symptoms in the narcoleptic syndrome. J Sleep Res 1994;3:52-9.

[26] Keltikangas-Jarvinen L, Rimon R. Rimon's Brief Depression Scale, a rapid method for screening depression. Psychol Rep 1987;60:111-19.

[27] Regional Office for Europe WHO. Well-Being measures in primary health care: The DepCare Project. Consensus meeting, Stockholm, 1998.

[28] Pizza F, Franceschini C, Peltola H, et al. Clinical and polysomnographic course of childhood narcolepsy with cataplexy. Brain 2013;136:3787-95.

[29] Black J, Houghton WC. Sodium oxybate improves excessive daytime sleepiness in narcolepsy. Sleep 2006;29:939-46.

[30] Baumann CR, Khatami R, Werth E, et al. Hypocretin (orexin) deficiency predicts severe objective excessive daytime sleepiness in narcolepsy with cataplexy. J Neurol Neurosurg Psychiatry 2006;77:402-4.

[31] Dauvilliers Y, Bassetti C, Lammers GJ, et al. Pitolisant versus placebo or modafinil in patients with narcolepsy: a double-blind, randomised trial. Lancet Neurol 2013;12:1068-75.

[32] Snyder CF, Aaronson NK. Use of patient-reported outcomes in clinical practice. Lancet 2009;374(9687):369-70. 